\title{
SEASONAL EFFECTS IN THE ELIMINATION OF TRACHOMA
}

\author{
DAVID C. LEE, JAYA D. CHIDAMBARAM, TRAVIS C. PORCO, AND THOMAS M. LIETMAN \\ Francis I. Proctor Foundation, Department of Ophthalmology, Department of Epidemiology and Biostatistics, and Institute for \\ Global Health, University of California, San Francisco, California; California Department of Health Services, Berkeley, California
}

\begin{abstract}
The World Health Organization currently recommends annual mass antibiotic treatment to eliminate the ocular chlamydia that cause blinding trachoma. Active trachoma is believed to be seasonal in many areas of the world, and the optimal season in which to treat has not as yet been established. Here we use mathematical models of disease transmission to demonstrate that ideally, treatment should be administered before the low season to have the greatest chance of locally eliminating infection.
\end{abstract}

\section{INTRODUCTION}

Trachoma is the leading cause of infectious blindness worldwide. Although it has disappeared throughout much of the world, it still affects more than 150 million people in developing countries. ${ }^{1}$ The World Health Organization (WHO), along with non-governmental organizations and public health services, has launched a major initiative to eliminate the disease. ${ }^{2}$ A key element in this effort has been the annual mass administration of systemic antibiotics. Since it is difficult to identify which individuals harbor the ocular chlamydial infection that causes trachoma, antibiotics are often distributed simultaneously to an entire community. Mass distribution of azithromycin has been surprisingly successful in reducing infection. ${ }^{3,4}$ However, the seasonal timing of treatments has not been addressed. In this study, we use mathematical models of trachoma transmission to determine which season annual mass antibiotic treatments should be administered to most effectively eliminate trachoma.

\section{METHODS}

We model the prevalence of ocular chlamydial infection in a community over time in two different ways: with differential equations, where the results at any time-point are completely dependent on the results at previous time-points (deterministic transmission model), and with simulations, where the effects of chance are also included (stochastic transmission model).

Deterministic transmission model. The transmission of ocular chlamydia within a community can be described by the nonlinear ordinary differential equation shown in Equation $1 .^{5}$

$$
\frac{d P}{d t}=\beta(1-P) P-\gamma P
$$

The prevalence, $P$, changes over time, $t$, at a rate equal to the rate of new infections minus the rate of recovery from existing infections. The rate of new infections is dependent on the rate of contact between individuals that can result in transmission $(\beta)$ and the probability that one of the two individuals who meet is susceptible $(1-P)$ and the other infectious $(P)$. A decrease in prevalence occurs when an infected individual recovers (at a rate $\gamma$ ) and depends on the prevalence of infected individuals. We assume that ocular chlamydial infection does not affect mortality. The general solution for this differential equation is a logistic equation. ${ }^{5}$

Annual treatment is modeled by decreasing the prevalence by an amount equal to the treatment coverage of the population times the efficacy of the antibiotic in an individual. ${ }^{6} \mathrm{We}$ assume an average efficacy of $95 \%$ based on studies of macrolides in ocular and genital infections. ${ }^{6}$ While $60-95 \%$ coverage has been achieved in various settings, we use an average coverage of $80 \%$, the target of the WHO for treatment programs. $^{3,7,8}$ We set values of $\beta$ and $\gamma$ to be 0.2 and 0.1 per month respectively, consistent with infection returning posttreatment at an exponential rate of $10 \%$ per month, the rate found empirically in a recent study of 24 Ethiopian villages. ${ }^{4}$

Seasonal effects. To model a seasonal effect, the transmission parameter $(\beta)$ is varied over time so that the equilibrium prevalence fluctuates in a sinusoidal manner over a year. Equation 2 shows the resulting modified transmission parameter, $\beta^{\prime}$, with its phase determined by an offset measured in months.

$$
\beta^{\prime}=\beta\left[1+\cos \frac{2 \pi(t+\text { monthly offset })}{12}\right]
$$

Stochastic transmission model. Since a deterministic model does not account for the chance effects that may result in local extinction or fadeout of infection in small communities, ${ }^{9}$ we also perform simulations with a stochastic model using the same parameters as the deterministic model. We assume an effective community size of 100 individuals because there are approximately 100 children $\leq 10$ years old per community in previously studied trachoma-endemic areas of Ethiopia, the age group that carries the greatest burden of infection. ${ }^{4}$ Using standard event-driven simulation techniques, ${ }^{10}$ the rates at which individuals become infected or recover from infection (i.e., disease state changes) are used to determine the time between such events occurring to individuals within the village. ${ }^{11,12}$ When the next stochastic event for a village would not occur until after the next scheduled periodic treatment, we simulate the institution of treatment, and then compute the times of further stochastic events representing disease state changes for individuals in the village using the posttreatment parameter values. Results from 5 sets of 2,000 simulated communities were tabulated at 8 evenly spaced times of the year. The time it took to achieve elimination in $75 \%, 90 \%$, or $95 \%$ of the villages was determined.

\section{RESULTS}

The deterministic model shows that the repeated administration of antibiotics can progressively reduce infection so that it approaches elimination, given adequate antibiotic efficacy and coverage. The effect of treatment without season- 
ality is depicted by the gray shading in Figure 1. Prior to treatment, the prevalence of infection in the deterministic seasonal effect model fluctuates between approximately $40 \%$ and $60 \%$. Similarly, the prevalence continues to fluctuate after treatments, depending on the timing of treatment. Figure 1 shows the course of infection with rounds of annual treatment starting at four evenly spaced times of year. The two curves in Figure 1A display the prevalence of infection if annual treatment were to be given three months before the high season or three months before the low season. Figure 1B shows the effect of treating at the peak of the high season or the peak of the low season.

For the stochastic model, the average pre-treatment prevalence fluctuates over the year as depicted by the gray shading in Figure 2. Treating three months before the low season results in elimination in the shortest period of time. Treating three months before the high season is the least effective. This seasonal pattern is found whether we monitor the length of time for elimination in $75 \%, 90 \%$, or $95 \%$ of the communities (Figure 2). The 95\% confidence interval for the average time until elimination in a certain percentage of villages at any time-point ranged from \pm 2 months to \pm 5 months.

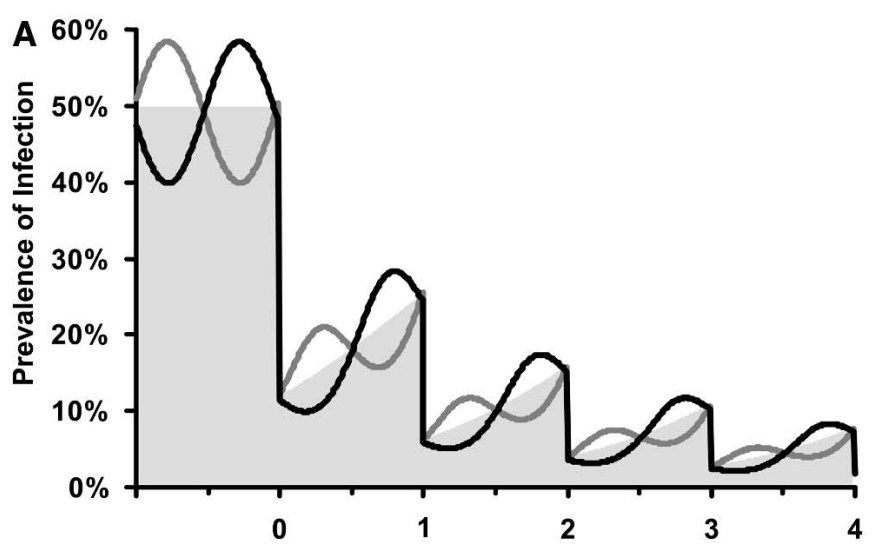

Time After Initial Treatment (Years)

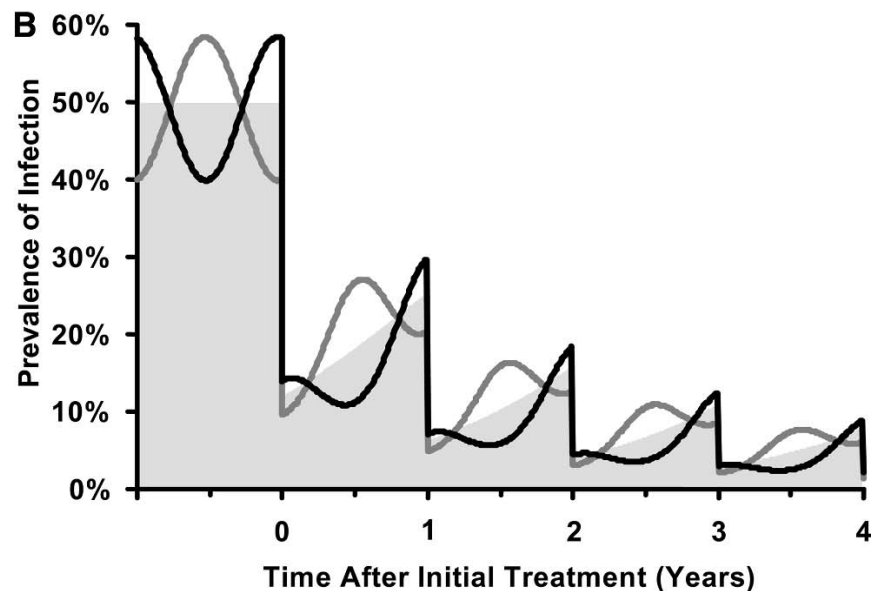

FiguRE 1. Results from a deterministic seasonal model of trachoma transmission. Parameters include $95 \%$ antibiotic efficacy, $80 \%$ treatment coverage, exponential infectious rate of $10 \%$ per month, and average pre-treatment prevalence of $50 \%$. Prevalence is shown without a seasonal effect for comparison (gray shading). A, Treatment is repeated annually three months before the low season (black line) or three months before the high season (gray line). B, Treatment is repeated annually at the peak of the high season (black line) or at the trough of the low season (gray line).

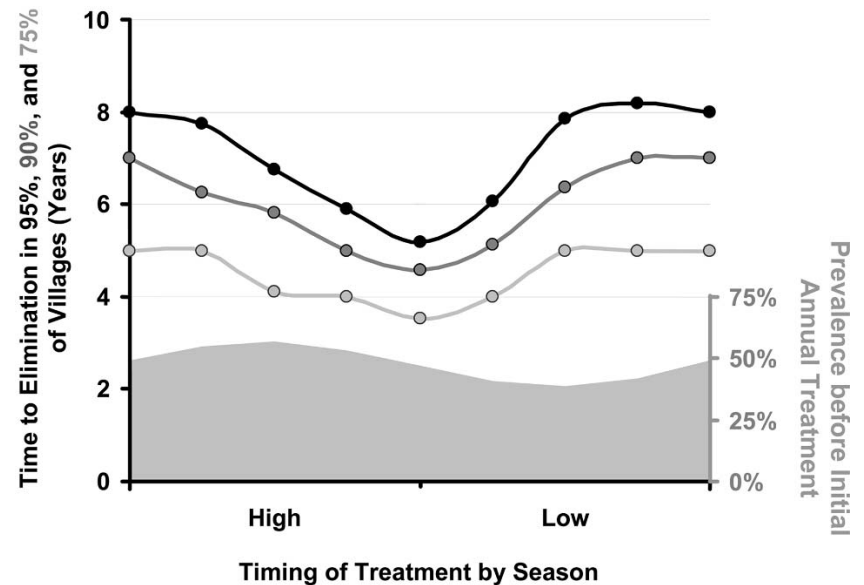

Figure 2. Results from a stochastic seasonal model of trachoma transmission. Prevalence by season prior to the first annual treatment (gray shading). Treatment duration as the time to elimination of infection in $95 \%$ (black line), $90 \%$ (dark gray line), and $75 \%$ (light gray line) of communities based on the averages of five sets of 2000 simulations at eight treatment time points (circles). Parameters include an average $95 \%$ antibiotic efficacy, $80 \%$ treatment coverage, exponential infectious rate of $10 \%$ per month, average pre-treatment prevalence of $50 \%$, and simulations of an effective population size of 100 individuals per village.

A sensitivity analysis was performed by adjusting treatment coverage by $\pm 10 \%$, transmission parameters by $\pm 50 \%$, and the amplitude of seasonality by $\pm 50 \%$. These scenarios resulted in different times for infection to be eliminated in villages. However, the minimum duration of treatment required to eliminate infection in $75 \%, 90 \%$, or $95 \%$ of the villages was still found at the same time-point: three months before the trough of the low season.

\section{DISCUSSION}

The prevalence of trachoma has been shown to vary by season in many areas of the world, including Morocco, Tunisia, Egypt, and Nepal. ${ }^{7,13,14}$ This may be due to the fluctuation in fly density or water availability because flies are thought to contribute to transmission and better hygiene may decrease transmission. ${ }^{15}$ Currently, it is not clear whether the annual mass antibiotic treatments recommended by the WHO would best be administered in the high season or the low season. One relatively short-term study in Nepal randomized six villages to treatment in the spring or the fall; in this area, a seasonal effect was identified, and the clinical prevalence of trachoma was $20 \%$ higher in the spring $(P<0.001)$. No significant difference in treatment season was found one year after treatment $(P=0.61){ }^{16}$ However this study had the power only to detect a relatively large effect and only compared treatments given at two times of the year. Further empirical studies have not been performed, in part due to the high cost of monitoring a larger number of villages at multiple points in the year.

Here, we used mathematical models to evaluate the optimal time of the year to perform mass antibiotic treatments. A deterministic model suggests that the time course of infection returning into a community would be different within a year depending on the treatment season (Figure 1). However, 
there are no clear year-to-year differences, even with a relatively strong seasonal effect. Unlike deterministic models, stochastic models can explicitly account for chance effects and the local elimination of infection in a community. Stochastic simulations demonstrate that the optimal time to treat to eliminate infection in a community is three months before the trough of the low season (three months after the peak of the high season; Figure 2). This represents the time when transmission rates are the lowest and offers the highest probability of local elimination in communities of limited population sizes.

Even if the ideal time to treat is before the low season, it should be recognized that other considerations may play a greater role. For example in some areas, this time point may fall during seasons when access to remote villages may not be feasible, for example, during the monsoon. It may also be hard to attain high treatment coverage due to social reasons such as religious events and festivals. Furthermore, programs may not be able to concentrate all of their distribution efforts into a few critical months of the year. Since it is difficult to determine precisely when the high and low season actually occur, it may be difficult to structure a program with the necessary precision to take advantage of the effect observed in this study. However, mathematical models suggest that, other things being equal, we increase the probability of locally eliminating infection by treating after the high season and before the low season. Mathematical models may be of particular use in cases such as this where large-scale clinical trials will likely never be performed.

Received October 18, 2004. Accepted for publication November 29, 2004.

Acknowledgments: We thank Stephanie Costanza at the Proctor Foundation for her invaluable help with this study.

Financial support: This work was supported by That Man May See, Research to Prevent Blindness, the National Institute of Allergy and Infectious Diseases (grant R21 AI55752-01), and the National Eye Institute (grant U10 EY16214-01).

Authors' addresses: David C. Lee, F. I. Proctor Foundation, 95 Kirkham Street, University of California, San Francisco, San Francisco, CA 94143-0944, Telephone: 415-502-2662, Fax: 415-476-0527, E-mail: dleeatfas@gmail.com. Jaya D. Chidambaram, F. I. Proctor Foundation, 95 Kirkham Street, Room 306, University of California, San Francisco, San Francisco, CA 94143-0944, Telephone: 415-5142163, Fax: 415-476-0527, E-mail: jaya@itsa.ucsf.edu. Travis C. Porco, Senior Epidemiologist, California Department of Health Services, Tuberculosis Control Branch Surveillance and Epidemiology Section, 2151 Berkeley Way, Room 608, Berkeley, CA 94704, Telephone: 510-540-3516, Fax: 510-540-2062, E-mail: TPorco@dhs.ca.gov. Thomas M. Lietman, World Health Organization Collaborating Center for the Prevention of Blindness, F. I. Proctor Foundation, 95 Kirkham Street, Room 307, University of California San Francisco, San Fran- cisco, CA 94143-0944, Telephone: 415-502-2662, Fax: 415-476-0527, E-mail: tml@itsa.ucsf.edu.

\section{REFERENCES}

1. Thylefors B, Négrel AD, Pararajasegaram R, Dadzie KY, 1995. Global data on blindness. Bull World Health Organ 73: 115121.

2. WHO, 1997. Report of the First Meeting of the WHO Alliance for the Global Elimination of Trachoma. Geneva: World Health Organization.

3. Schachter J, West SK, Mabey D, Dawson CR, Bobo L, Bailey R, Vitale S, Quinn TC, Sheta A, Sallam S, Mkocha H, Faal H, 1999. Azithromycin in control of trachoma. Lancet 354: 630 635.

4. Melese M, Chidambaram JD, Alemayehu W, Lee DC, Yi EH, Cevallos V, Zhou Z, Donnellan C, Saidel M, Whitcher JP, Gaynor BD, Lietman TM, 2004. Feasibility of eliminating ocular Chlamydia trachomatis with repeat mass antibiotic treatments. JAMA 292: 721-725.

5. Anderson R, May R, 1991. Infectious Diseases of Humans: Dynamics and Control. Oxford, United Kingdom: Oxford University Press.

6. Lietman T, Porco T, Dawson C, Blower S, 1999. Global elimination of trachoma: how frequently should we administer mass chemotherapy? Nature Med 5: 572-576.

7. Holm SO, Jha HC, Bhatta RC, Chaudhary JS, Thapa BB, Davis D, Pokhrel RP, Yinghui M, Zegans M, Schachter J, Frick KD, Tapert L, Lietman TM, 2001. Comparison of two azithromycin distribution strategies for controlling trachoma in Nepal. Bull World Health Organ 79: 194-200.

8. Lynch M, West S, Munoz B, Frick KD, Mkocha HA, 2003. Azithromycin treatment coverage in Tanzanian children using community volunteers. Ophthalmic Epidemiol 10: 167-175.

9. Bartlett MS, 1956. Deterministic and Stochastic Models for Recurrent Epidemics. Berkeley, CA: Proceedings of the Third Berkeley Symposium on Mathematical Statistics and Probability 4: 81-110.

10. Bratley P, Fox BL, Schrage LE, 1987. A Guide to Simulation. New York: Springer-Verlag.

11. Bailey N, 1957. The Mathematical Theory of Epidemics. London: Griffin.

12. Lloyd AL, 2004. Estimating variability in models for recurrent epidemics: assessing the use of moment closure techniques. Theor Popul Biol 65: 49-65.

13. Reinhard J, Weber A, Nizetic B, Kupka K, Maxwell-Lyons F, 1968. Studies in the epidemiology and control of seasonal conjunctivitis and trachoma in southern Morocco. Bull World Health Organ 29: 497-545.

14. da Cruz L, Dadour IR, McAllister IL, Jackson A, Isaacs T, 2002. Seasonal variation in trachoma and bush flies in north-western Australian Aboriginal communities. Clin Exp Ophthalmol 30: 80-83.

15. Emerson PM, Cairncross S, Bailey RL, Mabey DC, 2000. Review of the evidence base for the ' $F$ ' and ' $E$ ' components of the SAFE strategy for trachoma control. Trop Med Int Health 5: 515-527.

16. Jha H, Chaudary J, Bhatta R, Miao Y, Osaki-Holm S, Gaynor B, Zegans M, Bird M, Yi E, Holbrook K, Whitcher JP, Lietman $\mathrm{T}, 2002$. Disappearance of trachoma in western Nepal. Clinical Infectious Diseases 35: 765-768. 